\title{
Cultural differences in preferences for facial coloration
}

Chengyang Han ${ }^{\mathrm{a}}$, Hongyi Wang ${ }^{\mathrm{a}}$, Amanda C. Hahn ${ }^{\mathrm{a}, \mathrm{b}}$, Claire I. Fisher ${ }^{\mathrm{a}}$, Michal Kandrik $^{\mathrm{a}}$, Vanessa Fasolt ${ }^{\mathrm{a}}$, Danielle K. Morrison ${ }^{\mathrm{a}}$, Anthony J. Lee ${ }^{\mathrm{a}}$, Iris J. Holzleitner ${ }^{\mathrm{a}}$, Lisa M. DeBruine ${ }^{a}$, and Benedict C. Jones ${ }^{a}$

a. Institute of Neuroscience and Psychology, University of Glasgow, Glasgow, Scotland, United Kingdom.

b. Amanda C. Hahn is now at Department of Psychology, Humboldt State University, Arcate, CA, United States.

This research was supported by European Research Council Grant OCMATE to BCJ

Correspondence concerning this article should be address to Chengyang Han, Institute of Neuroscience and Psychology, University of Glasgow

58 Hillhead Street, Glasgow, UK, G12 8QB. E-mail: c.han.1@research.gla.ac.uk

Word count: 3031

Accepted refereed manuscript of: Han C, Wang H, Hahn A, Fisher C, Kandrik M, Fasolt V, Morrison D, Lee AJ, Holzleitner IJ, DeBruine LM \& Jones BC (2018) Cultural differences in preferences for facial coloration. Evolution and Human Behavior, 39 (2), pp. 154-159. DOI: https://doi.org/10.1016/j.evolhumbehav.2017.11.005

(c) 2017, Elsevier. Licensed under the Creative Commons Attribution-NonCommercialNoDerivatives 4.0 International http://creativecommons.org/licenses/by-nc-nd/4.0/ 


\title{
Cultural differences in preferences for facial coloration
}

\author{
Abstract \\ Effects of facial coloration on facial attractiveness judgments are hypothesized to be \\ "universal" (i.e., similar across cultures). Cross-cultural similarity in facial color \\ preferences is a critical piece of evidence for this hypothesis. However, only two \\ studies have directly compared facial color preferences in two cultures. Both of those \\ studies reported that White UK and Black African participants showed similar \\ preferences for facial coloration. By contrast with the cross-cultural similarity \\ reported in those studies, here we show cultural differences in the effects of facial \\ coloration on Chinese and White UK participants' facial attractiveness judgments. \\ While Chinese participants preferred faces with decreased yellowness to faces with \\ increased yellowness, White UK participants preferred faces with increased \\ yellowness to faces with decreased yellowness. Chinese participants also \\ demonstrated weaker preferences for facial redness and stronger preferences for \\ facial lightness than did White UK participants. These results suggest that \\ preferences for facial coloration are not universal.
}

\section{Keywords}

attractiveness, cultural differences, face perception, health 


\section{Introduction}

Facial attractiveness judgments predict important social outcomes, such as decisions about who we choose to date, mate with, hire, and vote for (Langlois et al., 2000; Little, Jones, \& DeBruine, 2011; Rhodes, 2006). Consequently, identifying factors that influence facial attractiveness judgments can provide insight into the mechanisms and processes that underpin a potentially important driver of social behavior and outcomes (Langlois et al., 2000; Little et al., 2011; Rhodes, 2006). A first step in investigating this issue is testing whether facial characteristics have similar effects on attractiveness judgments in different cultures (Langlois et al., 2000; Little et al., 2011; Rhodes, 2006). Indeed, many researchers have reported "universal" preferences for aspects of face shape (see Little et al., 2011 and Rhodes, 2006 for reviews).

Effects of skin characteristics on facial attractiveness judgments are at least as large as (if not larger than) those of shape characteristics (Stephen et al., 2012; Said \& Todorov, 2011; Scott, Pound, Stephen, Clark, \& Penton-Voak, 2010; Torrance, Wincenciak, Hahn, DeBruine, \& Jones, 2014). Studies have reported that independently increasing yellowness, lightness, or redness in face images increases both their attractiveness and perceived health (Fisher, Hahn, DeBruine, \& Jones, 2014; Kandrik et al., 2017; Stephen, Law Smith, Stirrat, \& Perrett, 2009a; Stephen, Coetzee, Law Smith, \& Perrett, 2009b; Stephen, Coetzee, \& Perrett, 2011; see also Lefevre et al., 2013). These preferences for facial coloration are independent of possible effects of facial cues to ethnicity. Moreover, because facial coloration is linked to both oxygenated blood and diet (Stephen et al., 2009b; Whitehead et al., 2012), preferences for facial coloration are hypothesized to reflect adaptations that 
function to identify individuals in good physical health (Stephen et al., 2011, 2012). However, evidence that facial coloration is associated with measures of susceptibility to infectious diseases is equivocal (Foo, Rhodes, \& Simmons, 2017; Foo, Simmons, \& Rhodes, 2017; Henderson et al., 2017; Phalane et al., 2017).

Because facial coloration is hypothesized to be a valid health cue (Stephen et al., 2011,2012 ) and aversions to cues of poor health are hypothesized to occur across cultures (Langlois et al., 2000), some researchers have predicted that the effects of facial coloration on attractiveness judgments and health perceptions will be universal (Stephen et al., 2011, 2012). However, only two studies have directly compared facial color preferences in two cultures (Coetzee, Greeff, Stephen, \& Perrett, 2014; Stephen et al., 2012). Coetzee, Greeff, Stephen, and Perrett (2014) and Stephen et al. (2012) reported similar correlations between facial coloration and attractiveness in White UK and Black African participants. Studies investigating relationships between facial coloration and attractiveness judgments in either White UK or Black African samples have also reported similar preferences (e.g., Coetzee et al., 2012).

If preferences for facial coloration are similar across cultures, as has been claimed, then, cross-cultural similarity in facial color preferences should be evident in crosscultural comparisons other than those reported by Coetzee, Greeff, Stephen, and Perrett (2014) and Stephen et al. (2012). To investigate this issue, we compared Chinese and White UK participants' attractiveness judgments of faces that had been independently manipulated in global yellowness, lightness, and redness.

\section{Methods}




\subsection{Participants}

In total, 196 participants took part in the study. These included 52 White UK men $\left(M_{\text {age }}=22.83\right.$ years, $S D=5.55$ years $)$ and 49 White UK women $\left(M_{\text {age }}=21.82\right.$ years, $S D=3.64$ years), all of whom were born and resided in the UK. They also included 48 Chinese men $\left(M_{\text {age }}=24.47\right.$ years, $S D=3.64$ years $)$ and 47 Chinese women $\left(M_{\text {age }}\right.$ $=24.00$ years, $S D=2.75$ years), all of whom were born in China, but currently resided in the UK (mean number of years in the UK $=1.06$ years, $S D=0.95$ years).

\subsection{Stimuli}

First, full-face photographs of 5 young adult White UK men, 5 young adult White UK women, 5 young adult Chinese men, and 5 young adult Chinese women were taken under standardized photographic conditions. All individuals photographed were between 18 and 23 years old and were students at the University of Glasgow.

Photographs were taken in a small windowless room against a constant background, under standardized diffuse lighting conditions, and participants were instructed to pose with a neutral expression. Camera-to-head distance and camera settings were held constant. Photographs were taken using a Nikon D300S digital camera and a GretagMacbeth 24-square ColorChecker chart was included in each image for use in color calibration. These face images were color calibrated using a least-squares transform from an 11-expression polynomial expansion developed to standardize color information across images (Hong, Luo, \& Rhodes, 2001) and standardized on pupil positions.

CIELab color space (Commission Internationale de L'Éclairage, 1976) is modeled on the human visual system and consists of three independent color axes: yellow $\left(b^{*}\right)$, 
lightness $\left(L^{*}\right)$, and red $\left(a^{*}\right)$. We independently manipulated global coloration in the face images along each of these axes. Using methods described in Stephen et al. (2009a), we created six versions of each original face: One in which yellow was increased by three units, one in which yellow was decreased by three units, one in which lightness was increased by three units, one in which lightness was decreased by three units, one in which red was increased by three units, and one in which red was decreased by three units. Importantly, these manipulations only affect the manipulated color dimension (e.g., altering redness does not affect yellowness or lightness) and do not affect shape information (Stephen et al., 2009a). This technique for manipulating color information in faces has also been used in many other previous studies (e.g., Fisher et al., 2014; Kandrik et al., 2017; Stephen et al., 2011; Whitehead et al., 2012). These color manipulations, in which color values were increased or decreased by 3 units, are within the normal range of coloration for White UK (Whitehead et al., 2012) and Malaysian Chinese (Tan et al., 2017) adult faces. We manipulated facial color by $+/-3$ units because we have previously shown that this extent of manipulation produces preferences for facial coloration in White UK participants that are similar to those reported in other studies using White UK faces (Fisher et al., 2014). This process created sixty pairs of faces, where each pair consisted of a version of an identity in which color values on one color axis had been increased by 3 units and another version of the same identity in which color values on that same color axis had been decreased by 3 units. Images were masked so that hairstyle and clothing were not visible. Example stimuli are shown in Figure 1. 


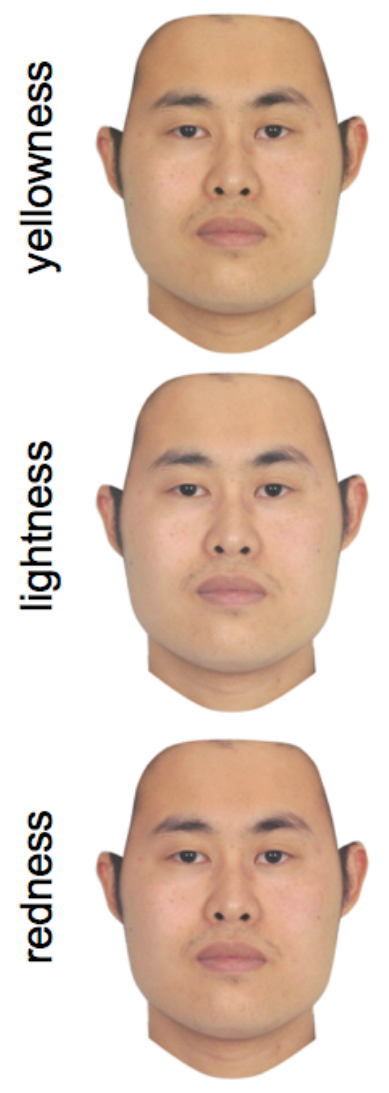

increased
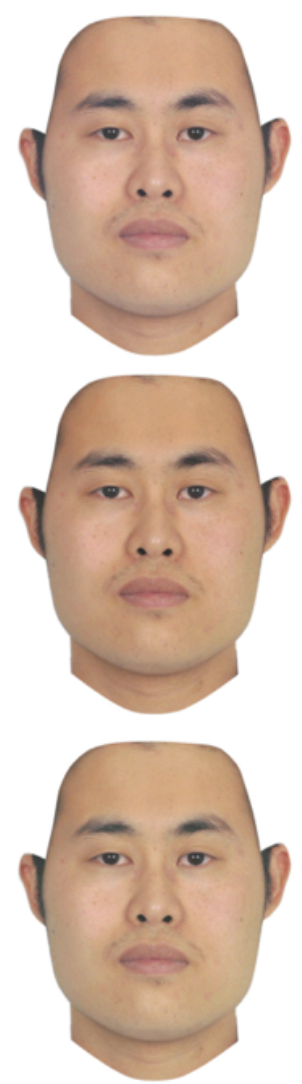

decreased
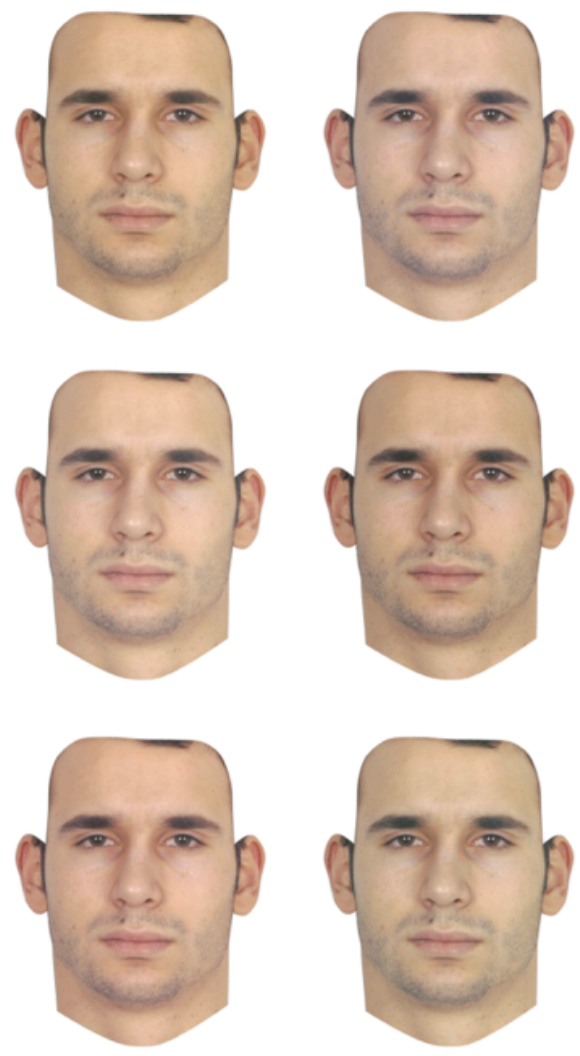

increased

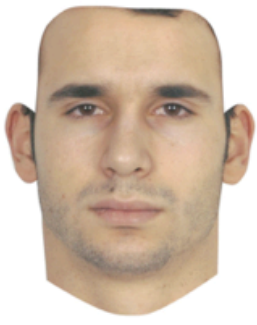

decreased

Figure 1. Examples of Chinese and White UK male face stimuli used in our study. The image on the left in each pair has increased color values and the image on the right in each pair has decreased color values. Images were independently manipulated in yellowness (top row), lightness (middle row), and redness (bottom row).

\subsection{Procedure}

The sixty pairs of faces were presented to participants using a two-alternative forced choice paradigm, in which participants were instructed to click on the face that they thought was the more attractive in each pair. In a separate block of trials, participants repeated this task, this time indicating which face in each pair looked healthier, rather than more attractive. These testing procedures control for the effects of prototypicality (i.e., averageness) of facial coloration on attractiveness judgments and health perceptions and have been used in previous work on facial color 
preferences (e.g., Kandrik et al., 2017). The order of the attractiveness-judgment and health-judgment tasks was fully randomized. Trial order and the side of the screen on which any given image was shown were fully randomized between participants. The screen was calibrated using an xRite i1 Display Pro colorimeter prior to testing.

\section{Results}

Responses on the attractiveness-judgment task were analyzed using mixed binary logistic regression analyses in R v3.3.2 (R Core Team, 2016) with Ime4 v1.1-12 (Bates, Maechler, Bolker, \& Walker, 2015). Preferences for facial yellowness, lightness, and redness were analyzed in separate models. In each model, the dependent variable was binary choice $(0=$ chose version of face with decreased color values, 1 = chose version of face with increased color values). Independent variables were sex of face (male, female), ethnicity of face (White UK, Chinese), sex of participant (male, female), and ethnicity of participant (White UK, Chinese), which were effect coded. For the sex of face and sex of participant variables, -0.5 corresponded to female and +0.5 corresponded to male. For the ethnicity of face and ethnicity of participant variables, -0.5 corresponded to White UK and +0.5 corresponded to Chinese. Each model specified random intercepts for participant and face. Random slopes were specified maximally, following Barr, Levy, Scheepers, and Tily (2013) and Barr (2013). Note that this type of analysis takes into account variation in the effects of the color manipulations across stimuli items (in this study, individual faces, Barr et al., 2013). Formulae and outputs for each model are given in our Supplemental Information, along with analysis scripts. Data are publicly available at osf.io/etxvu/. 


\subsection{Attractiveness judgments and facial yellowness}

The effects of facial yellowness on attractiveness judgments are summarized in Figure 2. The intercept was significant (beta $=-0.30, z=-2.40, p=.017$ ), indicating that, on average, participants preferred faces with decreased yellowness over those with increased yellowness. This was qualified by a main effect of ethnicity of participant (beta $=-1.48, z=-8.11, p<.001)$, indicating that Chinese and White UK participants differed significantly in the strength of their preferences for facial yellowness. Follow-up analyses indicated that White UK participants preferred versions of faces with increased yellowness over versions with decreased yellowness (intercept: beta $=0.45, z=2.72, p=.007$ ), but Chinese participants preferred versions of faces with decreased yellowness over versions with increased yellowness (intercept: beta $=-1.01, z=-7.05, p<.001$ ).

Our initial analysis also revealed significant main effects of sex of face (beta $=0.40$, $z=2.14, p=.032)$ and sex of participant (beta $=0.44, z=2.42, p=.016)$. These main effects indicated that preferences for facial yellowness were stronger for judgments of male faces than female faces and stronger for judgments by male participants than female participants. A significant interaction between ethnicity of participant and sex of participant (beta $=0.80, z=2.22, p=.027$ ) indicated that the effect of ethnicity of participant on preferences for facial yellowness was larger for female than male participants. No other effects were significant (all absolute betas < 0.51 , all absolute $z s<1.62$, all $p s>.10$ ). 


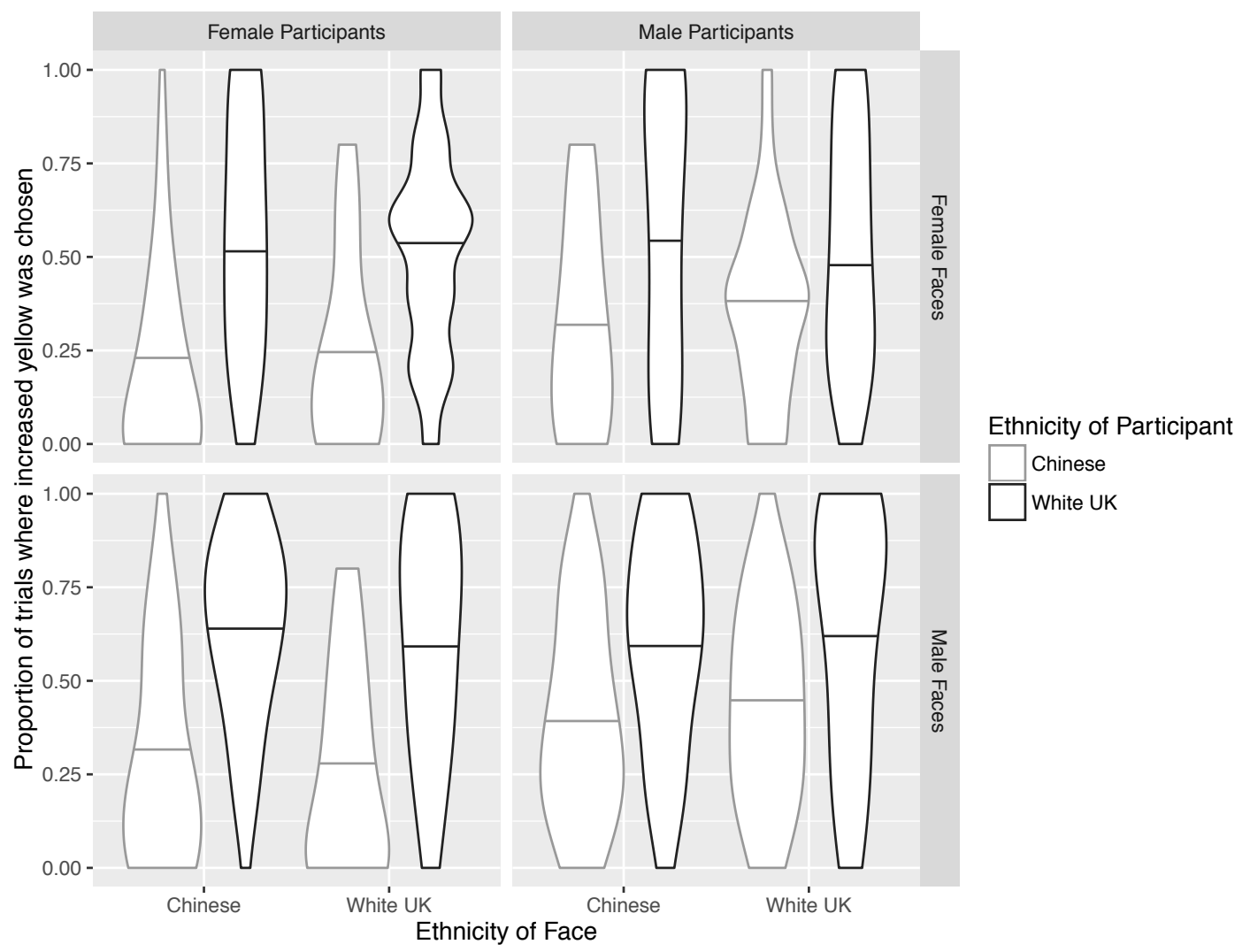

Figure 2. Attractiveness judgments and facial yellowness. Violin plots showing the distribution of Chinese and White UK male and female participants' responses for Chinese and White UK male and female faces manipulated in yellowness. The lines indicate medians and 0.5 on the $y$-axis equals chance.

\subsection{Attractiveness judgments and facial lightness}

The effects of facial lightness on attractiveness judgments are summarized in Figure 3. The intercept was significant (beta $=1.08, z=10.03, p<.001$ ), indicating that, on average, participants preferred faces with increased lightness over those with decreased lightness. This was qualified by a main effect of ethnicity of participant (beta $=1.53, z=7.55, p<.001)$, indicating that Chinese participants had stronger preferences for facial lightness than did White UK participants. A significant main effect of sex of face (beta $=-0.24, z=-2.23, p=.026)$ indicated that preferences for facial lightness were generally stronger for female faces than male faces. A 
significant interaction between sex of face and ethnicity of participant (beta $=0.35, z$ $=2.16, p=.031)$ indicated that the effect of sex of face on preferences for lightness was larger among White UK than Chinese participants. No other effects were significant (all absolute betas $<0.67$, all absolute $z s<1.63$, all $p s>.05$ ).

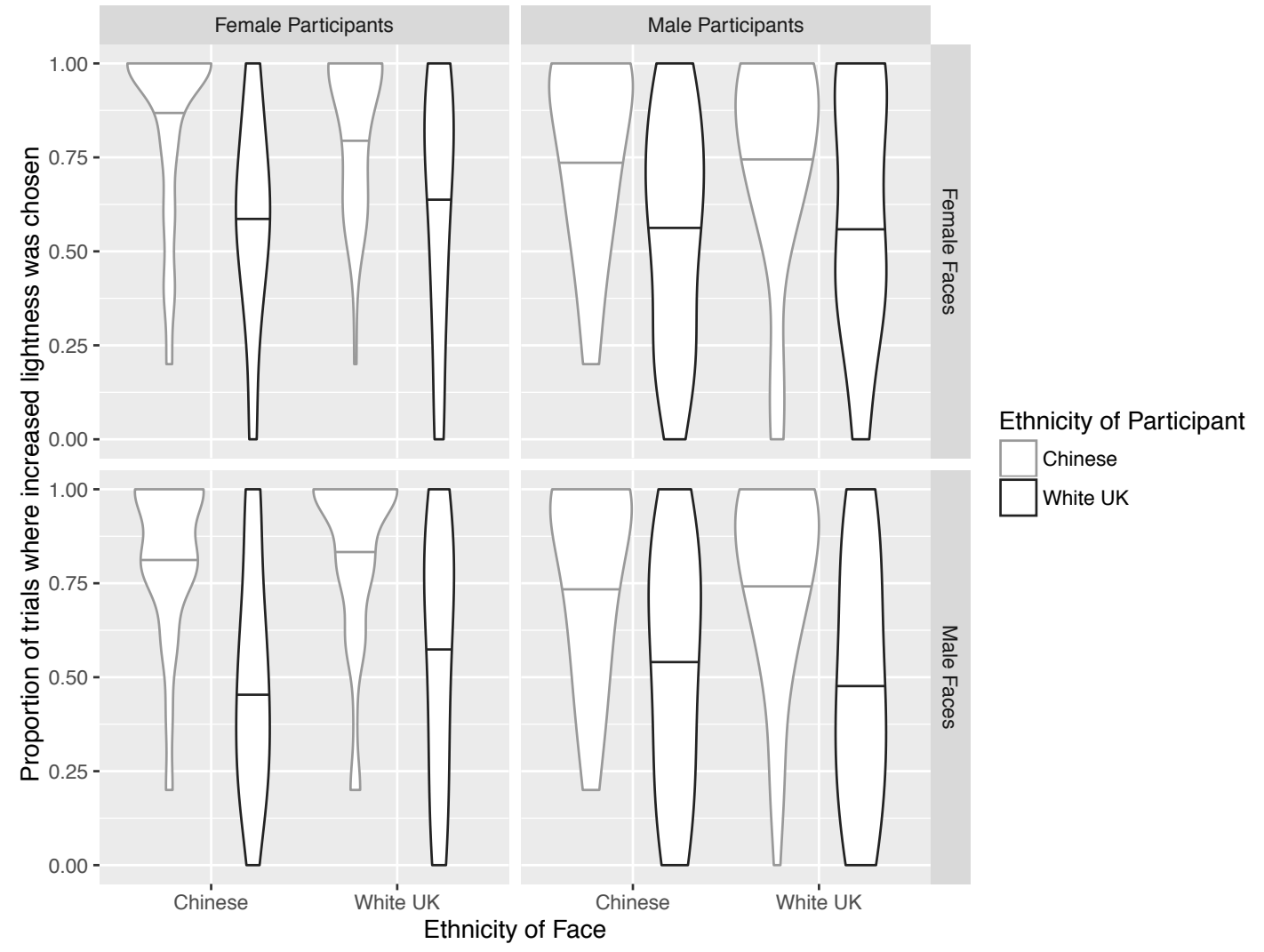

Figure 3. Attractiveness judgments and facial lightness. Violin plots showing the distribution of Chinese and White UK male and female participants' responses for Chinese and White UK male and female faces manipulated in lightness. The lines indicate medians and 0.5 on the $y$-axis equals chance.

\subsection{Attractiveness judgments and facial redness}

The effects of facial redness on attractiveness judgments are summarized in Figure 4. The intercept was significant (beta $=1.64, z=9.53, p<.001)$, indicating that, on average, participants preferred faces with increased redness over those with decreased redness. This was qualified by a main effect of ethnicity of participant 
(beta $=-0.58, z=-2.66, p=.007)$, indicating that White UK participants had stronger preferences for redness than did Chinese participants. A significant main effect of sex of face (beta $=-0.61, z=-2.21, p=.027$ ) indicated that preferences for redness were stronger for judgments of female than male faces. There were also significant interactions between sex of face and sex of participant (beta $=0.54, z=3.05, p$ $=.002)$, between ethnicity of face and sex of face (beta $=-1.14, z=-2.04, p=.041)$, between ethnicity of face and ethnicity of participant (beta $=-0.35, z=-2.02, p$ $=.043)$. These two-way interactions suggested that the tendency to show stronger preferences for redness in female than male faces was greater in female than male participants, that the tendency to show stronger preferences for redness in female than male faces was greater in Chinese than White UK faces, and that the tendency for White UK participants to show stronger preferences for redness than did Chinese participants was greater for Chinese faces than White UK faces, respectively. The four-way interaction among sex of face, ethnicity of face, sex of participant, and ethnicity of participant was also significant (beta $=-1.63, z=-2.20, p=.028$ ). Figure 4 suggested that the four-way interaction reflected the effect of ethnicity of participant being particularly large when male participants judged Chinese male faces. No other effects were significant (all absolute betas $<0.25$, all absolute $z s<$ 1.14 , all $p s>.25)$. 


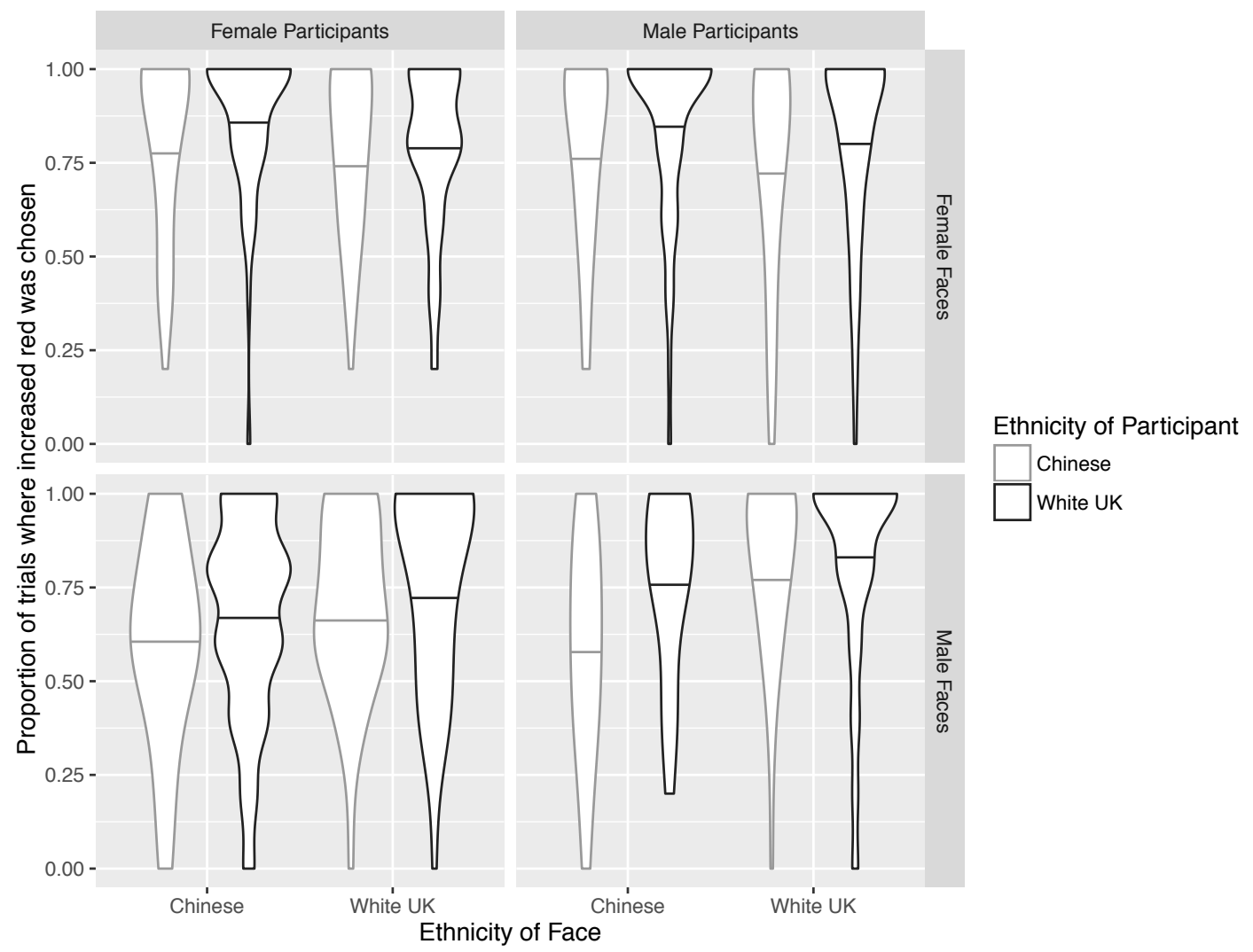

Figure 4. Attractiveness judgments and facial redness. Violin plots showing the distribution of Chinese and White UK male and female participants' responses for Chinese and White UK male and female faces manipulated in redness. The lines indicate medians and 0.5 on the $y$-axis equals chance.

\subsection{Effects of facial coloration on health perceptions}

Responses on the health-judgment task were analyzed in the same way as responses on the attractiveness-judgment task. Full results are given in our Supplemental Information. Health-judgment analyses showed the same effects of participant ethnicity to those reported for the attractiveness-judgment task, although the effect of participant ethnicity for health judgments of faces manipulated in redness was not significant $(p=.08)$. This similarity between our results for attractiveness and health judgments is consistent with previous research suggesting that facial attractiveness and health judgments are generally highly correlated (e.g., Jones et al., 2005). 
Repeating the analyses described above controlling for possible effects of participant age did not alter the pattern of significant effects.

\section{Discussion}

We compared Chinese and White UK participants' attractiveness judgments of faces that had been independently manipulated in global coloration. We show, for the first time, that Chinese participants preferred faces with decreased yellowness to faces with increased yellowness. By contrast, White UK participants preferred faces with increased yellowness to faces with decreased yellowness. These opposite preferences for facial yellowness in Chinese and White UK participants show that preferences for facial colorations are not necessarily universal, as some researchers have claimed (Stephen et al., 2011, 2012).

Our analyses also revealed cross-cultural differences in preferences for facial redness and lightness. Both Chinese and White UK participants preferred faces with increased redness and lightness over those with decreased redness and lightness. However, Chinese participants' preferences for facial redness were weaker than those observed for White UK participants and Chinese participants' preferences for facial lightness were stronger than those observed for White UK participants.

While our study is the first to compare facial color preferences in Chinese and White UK participants, studies have previously examined facial color preferences in White UK participants. Consistent with their results, the White participants in our study showed preferences for increased facial yellowness, redness, and lightness (Fisher 
et al., 2014; Kandrik et al., 2017; Stephen et al., 2009a, 2009b, 2011). The stronger preferences for facial lightness when judging female faces than when judging male faces that we observed in the current study has also been previously reported (Stephen et al., 2009a).

The effects of participant ethnicity on preferences for facial yellowness and lightness in our study were similar for judgments of both own-race and other-race faces. This is an important point, since it demonstrates that the differences in these facial color preferences between Chinese and White UK participants that we observed cannot be explained by hypothesized effects of stimulus ethnicity on preferences for facial coloration (Tan et al., 2017).

We also observed cross-cultural differences in the effects of facial coloration on health perceptions. In particular, facial yellowness had opposite effects on Chinese and White UK participants' health perceptions. This cross-cultural difference in the effects of yellowness on health perceptions is consistent with recent results suggesting that facial yellowness is not related to actual health. Fruit and vegetable consumption increases facial yellowness in both White UK and Malaysian participants (e.g., Foo, Rhodes, \& Simmons, 2017; Tan, Graf, Mitra, \& Stephen, 2017; Whitehead, Re, Xiao, Ozakinci, \& Perrett, 2012). While fruit and vegetable consumption is associated with high socioeconomic status in most developed countries, vegetable consumption is associated with low socioeconomic status in China (Wang, 2001). Consequently, we speculate that aversions to facial yellowness in Chinese participants could be due to facial yellowness functioning as a cue of low status. Alternatively, the cross-cultural differences in preferences for facial 
yellowness observed in the current study could reflect culture-specific associations between aspects of health and facial coloration, culture-specific stereotypes associated with subtle variation in ethnicity cues, or between-group differences in factors such as socioeconomic status. Further work is needed to investigate these issues.

In conclusion, our analyses of Chinese and White UK participants' preferences for facial coloration showed clear evidence for cross-cultural differences in preferences. These differences were particularly striking for facial yellowness, which had opposite effects on Chinese and White UK participants' face preferences. Importantly, these results directly challenge the hypothesis that facial color preferences are the same across cultures. 


\section{References}

Barr, D. J. (2013) Random effects structure for testing interactions in linear mixedeffects models. Frontiers in Psychology, 4, 328. doi:10.3389/fpsyg.2013.00328

Barr, D. J., Levy, R., Scheepers, C., \& Tily, H. J. (2013). Random effects structure for confirmatory hypothesis testing: Keep it maximal. Journal of Memory and Language, 68, 255-278.

Bates, D., Maechler, M., Bolker, B., \& Walker, S. (2015). Fitting linear mixed-effects models using Ime4. Journal of Statistical Software, 67, 1-48.

Coetzee, V., Faerber, S. J., Greeff, J. M., Lefevre, C. E., Re, D. E., Perrett, D. I. (2012). African perceptions of female attractiveness. PLoS ONE, 7, e48116

Coetzee, V., Greeff, J. M., Stephen, I. D., \& Perrett, D. I. (2014). Cross-cultural agreement in facial attractiveness preferences: the role of ethnicity and gender. PLOS ONE, 9, e99629.

Commission Internationale de L'Éclairage. (1976). CIE Colorimetry - Part 4: 1976 $L^{*} a^{*} b^{*}$ Color Space.

Fisher, C., Hahn, A. C., DeBruine, L. M. \& Jones, B. C. (2014). Integrating shape cues of adiposity and color information when judging facial health and attractiveness. Perception, 43, 499-508.

Foo, Y. Z., Rhodes, G., \& Simmons, L. W. (2017). The carotenoid beta-carotene enhances facial color, attractiveness and perceived health, but not actual health, in humans. Behavioral Ecology, 28, 570-578.

Foo, Y. Z., Simmons, L. W., \& Rhodes, G. (2017). Predictors of facial attractiveness and health in humans. Scientific Reports, 7, 39731. 
Henderson, A. J., Lasselin, J., Lekander, M., Olsson, M. J., Powis, S. J., Axelsson, J., \& Perrett, D. I. (2017). Skin colour changes during experimentally-induced sickness. Brain, Behavior \& Immunity, 60, 312-318.

Hong, G., Luo, M. R., \& Rhodes, P. A. (2001). A study of digital camera colorimetric characterization based on polynomial modeling. Color Research \& Application, $26,76-84$.

Jones, B. C., Perrett, D. I., Little, A. C., Boothroyd, L. G., Cornwell, R. E., Feinberg, D. R., Tiddeman, B. P., Whiten, S., Pitman, R. M., Hillier, S. G., Burt, D. M., Stirrat, M. R., Law Smith, M. J. \& Moore, F. R. (2005). Menstrual cycle, pregnancy and oral contraceptive use alter attraction to apparent health in faces. Proceedings of the Royal Society of London B, 272, 347-354.

Kandrik, M., Hahn, A. C., Fisher, C., Wincenciak, J., DeBruine, L. M. \& Jones, B. C. (2017). Are physiological and behavioral immune responses negatively correlated? Evidence from hormone-linked differences in men's face preferences. Hormones \& Behavior, 87, 57-61.

Langlois, J. H., Kalakanis, L., Rubenstein, A. J., Larson, A., Hallam, M., \& Smoot, M. (2000). Maxims or myths of beauty? A meta-analytic and theoretical review. Psychological bulletin, 126, 390-423.

Lefevre, C. E., Ewbank, M. P. Calder, A. J., von dem Hagen, E., Perrett, D. I. (2013). It is all in the face: carotenoid skin coloration loses attractiveness outside the face. Biology Letters, 9, 20130633.

Little, A. C., Jones, B. C., \& DeBruine, L. M. (2011). Facial attractiveness: evolutionary based research. Philosophical Transactions of the Royal Society B: Biological Sciences, 366, 1638-1659. 
Phalane, K. G., Tribe, C., Steel, H. C., Cholo, M. C. \& Coetzee, V. (2017). Facial appearance reveals immunity in African men. Scientific Reports, 7, 7443.

R Core Team (2016). R: A language and environment for statistical computing. R Foundation for Statistical Computing, Vienna, Austria.

Rhodes, G. (2006). The evolutionary psychology of facial beauty. Annual Review of Psychology, 57, 199-226.

Said, C. P., \& Todorov, A. (2011). A statistical model of facial attractiveness. Psychological Science, 22, 1183-90.

Scott, I. M. L., Pound, N., Stephen, I. D., Clark, A. P., \& Penton-Voak, I. S. (2010). Does masculinity matter? The contribution of masculine face shape to male attractiveness in humans. PLoS ONE, 5, e13585. doi:10.1371/journal.pone.0013585

Stephen, I. D., Law Smith, M. J., Stirrat, M. R., \& Perrett, D. I. (2009a). Facial skin coloration affects perceived health of human faces. International Journal of Primatology, 30, 845-857.

Stephen, I. D., Coetzee, V., Law Smith, M. J., \& Perrett, D. I. (2009b). Skin blood perfusion and oxygenation colour affect perceived human health. PLoS One, 4, e5083. doi:10.1371/journal.pone.0005083

Stephen, I. D., Coetzee, V., \& Perrett, D. I. (2011). Carotenoid and melanin pigment coloration affect perceived human health. Evolution \& Human Behavior, 32, 216-227.

Stephen, I. D., Scott, I. M., Coetzee, V., Pound, N., Perrett, D. I., \& Penton-Voak, I. S. (2012). Cross-cultural effects of color, but not morphological masculinity, on perceived attractiveness of men's faces. Evolution \& Human Behavior, 33, 260-267. 
Tan, K. W., Graf, B A., Mitra, R. S., \& Stephen, I. D. (2017). Impact of fresh fruit smoothie consumption on apparent health of Asian faces. Evolution \& Human Behavior, in press. http://doi.org/10.1016/j.evolhumbehav.2017.02.004

Torrance, J. S., Wincenciak, J., Hahn, A. C., DeBruine, L. M., \& Jones, B. C. (2014). The relative contributions of facial shape and surface information to perceptions of attractiveness and dominance. PloS ONE, 9, e104415. doi:10.1371/journal.pone. 0104415

Wang, Y. (2001). Cross-national comparison of childhood obesity: The epidemic and the relationship between obesity and socioeconomic status. International Journal of Epidemiology, 30, 1129-1136.

Whitehead, R. D., Re, D., Xiao, D., Ozakinci, G., \& Perrett, D. I. (2012). You are what you eat: Within-subject increases in fruit and vegetable consumption confer beneficial skin-color changes. PLoS ONE, 7, e32988.

doi:10.1371/journal.pone. 0032988 